\title{
Quantifying the uncertainty in a hyperelastic soft tissue model with stochastic parameters
}

\author{
Paul Hauseux ${ }^{\mathrm{a}}$, Jack S. Hale ${ }^{\mathrm{a}}$, S. Cotin ${ }^{\mathrm{b}}$, Stéphane P. A. Bordas ${ }^{\mathrm{a}, \mathrm{c}, *}$ \\ ${ }^{a}$ Institute of Computational Engineering, University of Luxembourg, 6 Avenue de la \\ Fonte, 4362 Esch-sur-Alzette, Luxembourg. \\ ${ }^{b}$ Team MIMESIS, Inria, Strasbourg, France. \\ ${ }^{c}$ Cardiff School of Engineering, Cardiff University, The Queens Building, The Parade, \\ Cardiff, Wales, CF24 $3 A A$, UK.
}

\begin{abstract}
We present a simple open-source semi-intrusive computational method to propagate uncertainties through hyperelastic models of soft tissues. The proposed method is up to two orders of magnitude faster than the standard Monte Carlo method. The material model of interest can be altered by adjusting few lines of (FEniCS) code. The method is able to (1) provide the user with statistical confidence intervals on quantities of practical interest, such as the displacement of a tumour or target site in an organ; (2) quantify the sensitivity of the response of the organ to the associated parameters of the material model. We exercise the approach on the determination of a confidence interval on the motion of a target in the brain. We also show that for the boundary conditions under consideration five parameters of the Ogden-Holzapfel-like model have negligible influence on the displacement of the target zone compared to the three most influential parameters. The benchmark problems and all associated data are made available as supplementary material.
\end{abstract}

Keywords: Keywords: Monte Carlo, uncertainty quantification, sensitivity analysis, hyperelastic models, brain deformation, UFL expressions, parallel computing, model selection.

\footnotetext{
${ }^{*}$ Corresponding author.

Email addresses: paul.hauseux@uni.lu (Paul Hauseux), mail@jackhale.co.uk (Jack S. Hale), stephane.cotin@inria.fr (S. Cotin), stephane.bordas@uni.lu (Stéphane P. A. Bordas)
} 


\section{Introduction}

Motivation and focus of the paper. Models and simulations are now commonly used to assist clinicians. As yet, there is however little work done to quantify the quality of the ensuing results. Errors in bioengineering simulations emanate either from the choice of the mathematical model (and its parameters: material properties, boundary conditions, etc.) used to describe the behavior of the system: this is known as model error; or from the numerical method used to solve the resulting problem: this is known as discretization error.

In this paper, we target a subclass of model errors. We focus on the simulation of soft tissue undergoing large deformations and address the problem of quantifying the effect of uncertainties on certain quantities of interest to the user e.g. average stress/displacement in a region. We assume that a material model has been chosen for the soft tissue under consideration and ask ourselves the question: "What is the effect of uncertainties on the knowledge of parameters on the accuracy of a predicted quantity of interest?"

Answering this question goes some way along the direction of quantifying modelling errors. A further step, which we do not consider here, but which is critical, is the selection of the best model given experimental data and a quantity of interest.

Concerning discretization error (associated with solving the mathematical problem numerically), the interested reader can refer to the abundant literature dealing with error estimation [1, 2] and to our recent paper proposing a real time error estimation and adaptive procedure for needle insertion [3].

Definitions. The word model 1 is to be understood as: A mathematical abstraction representing the behavior of a system amenable to being solved numerically (in a computer). For example, we will talk of constitutive models/material models which are mathematical abstractions relating the deformation of a body to the force required to cause this deformation. A geometrical model of a patient's anatomy is a set of parametric surfaces representing the boundary and internal structures of the patient's organs.

Challenges for computational medicine. There are several challenges for computational medicine which we review briefly. In order to make reliable predictions, a virtual representation of the patient must be generated

\footnotetext{
${ }^{1}$ When referring to other models, we will make the distinction clear, e.g. physical models, phantoms.
} 
from medical images. This is made difficult because of the lack of automatic segmentation methods for medical images [4, 5], although this is somewhat alleviated by the image as a model approach of K. Miller's group [6].

Once the images are segmented, digital representations of the geometries of the organ must be generated. If finite element methods (FEM) are used, this requires generating a mesh [7, 8], which can be very challenging, in particular within clinical time scales. Alternatives include implicit boundary definitions [9] or meshless methods [10].

Finally, once a geometrical model of the organ(s) under consideration is available, a material model must be chosen. This is probably the hardest hurdle to overcome because of the large variability in models (and their associated material properties) across patients. The suitability of the model, and the optimal parameters also depend on the age and lifestyle of the patient, environmental conditions, and many other factors that are difficult to factor in the simulations. Even in cases where the organ is externally accessible (like the skin) selecting the best material model and identifying the associated parameters is still non-trivial.

Uncertainties. This variability of human tissue makes it challenging to model. Moreover, acquiring data on human tissue is difficult because of obvious ethical considerations. Whilst it is possible and common to test samples in the laboratory to infer the suitability of a given material for engineering applications, in vivo testing is often impossible and always restricted when dealing with biological materials. Since cadaveric tissue behaves vastly differently from live tissue, acquiring data is all the more challenging.

This situation causes at least three difficulties:

- The models we develop are dependent on the patient under consideration. This is known as structural uncertainty;

- The parameters of these models are uncertain and patient/age/gender/lifestyle dependent. This is known as parametric uncertainty;

- Data on the patient of interest is usually sparse, of unknown quality and multi-modal (generated by different sources).

As it stands, the fundamental question of uncertainty and error quantification for clinically relevant quantities of interest remains largely unaddressed in computational medicine. Taking into account uncertainty in biomechanics models is a relatively young field and little work has been done in particular for soft-tissue with large deformation in a finite element context. 
The need for uncertainty quantification in biomechanics. Various applications exist in which numerical simulations are used to enhance medicine, e.g. for simulating pressure and flow propagation [11], for surgery simulation in real time [12, for soft tissue biomechanical modeling [13, 14] or to create and analyze dynamic simulations of movement [13, 14]. In some cases, due to insufficient experimental data available in certain areas of the body, simulations can improve understanding by replacing or complementing physical experiments [15].

In most of the above use cases, it is necessary to simulate the deformation of interacting organs. It is also often of importance to simulate the motion of a surgical target during the intervention, relative to its position in preoperative images. To do this, a material model suitable to the patient under consideration and its parameters are required, which causes significant difficulties. The choice of parameters (material properties, boundary conditions, loading, etc.) used in a numerical model is one of the main difficulties because of the variability in the results of material characterization. This is particularly true for the brain where differences can reach one to two orders of magnitude in published data. In [16] mechanical properties of brain tissue in tension are presented. The brain is very complex and anisotropic models are needed given that the mechanical response varies significantly depending on the loading modes: tension [17], shear [18] or compression [19. In the literature, a large number of hyperelastic constitutive models have been proposed to simulate the behavior of the brain. A comparison of hyperelastic constitutive models applicable to brain and fat tissues is given in [20]. In [21], the authors provide an approach to select the best model in relation to specific applications. The choice of the model and the parameters involved in the model are the main issues to address for the numerical simulation of soft tissue. Moreover some uncertainties are very difficult to determine because of inter-patient variability or too sparse and rare experiments. When safety-critical decisions must be made based on the output of simulations, being able to propagate and visualize the uncertainty due to material models and parameters is of key importance [22].

Uncertainty quantification in biomechanics. In deterministic modelling approaches of soft tissues, the constitutive material model is assumed known and the parameters of this model are deterministic. The results of simulations are also deterministic. For example, simulating the displacement of a target in the brain during brain shift [23] provides one deterministic value of displacement, say $3 \mathrm{~mm}$. In forward uncertainty quantification, a constitutive material model is also assumed a priori, but each material parameter 
of this model follows a given probability distribution, e.g. a normal distribution around a mean with a given standard deviation. The result of such a stochastic simulation is therefore also a probability distribution which can be characterized by statistical properties such as its mean, standard deviation or other "expected values" (see Eq. 2). For example, the result of the stochastic prediction of the displacement of a target in the brain would be a "normally distributed displacement with mean $3 \mathrm{~mm}$ and standard deviation $0.2 \mathrm{~mm}$ ". Given the fact that material parameters and models are so strongly variable for soft tissues, stochastic simulations seem more appropriate than deterministic simulations and offer the ability to provide the user with confidence intervals.

In spite of their attractiveness, stochastic methods are also much more computationally expensive. Indeed, one of the main approaches in stochastic modelling, known as the Monte Carlo method, is to run a (large) number of simulations for each value of the parameters and to compute the mean, standard deviations and other statistical characteristics of the solution by averaging. In other words, the Monte Carlo method is a direct numerical integration approach which transforms the integrals required to compute the statistical expectations into (usually large) discrete sums. As the parameters of the constitutive material model may vary over potentially very significant ranges, the domain of variation of each parameter has to be properly sampled to obtain representative results. This requirement to compute large sums makes the simulations much more expensive than deterministic simulations.

For large parameter sets and parameter ranges, methods were developed to accelerate the simulations by focusing the sampling of the parameter space where it is most important (advanced Monte Carlo methods). For example, the method called Importance Sampling generate from a distribution other than the original distribution of interest in order to reduce the variance of the estimator while preserving a convergence to the same estimator obtained by the regular Monte Carlo method. Note that even for a linear problem the deterministic solution with average parameters is different from the expected value (except for an additive functional ${ }^{2}$ ). Thus, to obtain the expected value of a stochastic differential equation, a statistical treatment is always needed.

The challenges of efficiently propagating these random variables and

\footnotetext{
${ }^{2}$ an additive problem is a linear problem with uncertainty only in the right-hand side: $K \cdot u(\omega)=f(\omega)$. For an additive problem $\mathrm{K}$ is deterministic and therefore does not depend on the random variable $\omega$ but the solution $u$ and the right-hand side $f$ depend on $\omega$.
} 
fields are therefore considerable because stochastic analyses require solving the numerical problem (via the finite element in this paper) sufficiently many times for the parameter space to be properly sampled. Monte Carlo methods [24] are popular because they are non-intrusive, i.e. they can be used with any standard piece of software able to solve the numerical problem for a given set of parameters. These methods are also relatively easy to implement, also in parallel because of the independence of the FE solutions for different values of the parameters. The convergence of Monte Carlo methods is guaranteed, independent of the stochastic dimension but slow (the error is inversely proportional to the square root of the number of realizations). Reducing computational expense is therefore critical.

An alternative to non-intrusive Monte Carlo methods are intrusive methods, such as stochastic Galerkin finite element methods (SGFEM) [25]. These methods lead to a unique but large-sized problem using a spectral approach. The numerical solution of this system provides directly an approximation of the probability density function (PDF) of the solution and then it is possible to evaluate the PDF of the quantity of interest. For example in mechanics, we find directly the PDF of the displacement $u$ and then we can evaluate the PDF of the stress field (a potential quantity of interest) which depends on $u$ and elastic constants. This approximation allows an a posteriori statistical treatment and thus unveils wider analysis possibilities than Monte Carlo methods. These methods are very efficient for linear PDEs with a small number of parameters (stochastic dimension), but are more complex to implement into existing software, in particular for non-linear PDEs due to their intrusive nature. We also note that it is possible to implement SGFEM in a partially intrusive manner with iterative solution techniques when models that can provide matrix-vector actions are available [26] and there are also new techniques for non-intrusive SGFEM methods that only require access to a deterministic residual [27].

The novelty of this paper. Most publications in biomechanics only use Monte Carlo simulations to propagate and quantify uncertainties in a finite element context, e.g., [28, 29]. An overview of stochastic analysis methods in biomechanics is given in [30]. Variations of Monte Carlo simulations are presented like Latin Hypercube Sampling (LHS) and the Importance Sampling Method (ISM) or also Response surface methods (RSMs) which are based on Monte Carlo simulations. In [31], a method for automatic modelling of boundary conditions in deformable anatomical structures is presented. The method 
is based on a statistical atlas which gathers data defining the connective structures attached to the organ of interest. A Markov Chain Monte Carlo sampler to draw deformation configurations from the posterior distribution is used in [32]. The method is based on a biomechanical finite element model which treats the prostate as an elastic material. They also demonstrate the importance that uncertainty information could have on neurosurgical decision making. Stochastic projection schemes for deterministic linear elliptic partial differential equations on random domains are proposed in 33 .

The focal point of this paper is the forward propagation of uncertainties and the reader can refer to [34, 35, 26] for a more detailed and formal introduction to stochastic modelling. More specifically, we present a stochastic finite element approach which we apply to brain deformation to uncover the relative importance of material parameters for a few hyperelastic constitutive material models for the brain. The coefficients of the models, used to define the hyperelastic strain energy density are assumed to follow known statistical distributions which would typically be provided by atlases, experiments, or live data acquired during the simulation as in data-driven modelling frameworks. We model uncertainties through random variables and correlated random fields [35]. We implement and test the required numerical solvers to propagate these uncertainties and, in turn, provide statistical solutions and confidence intervals on quantities of interest.

To reduce the error of the Monte Carlo approach, we develop an accelerated Monte Carlo estimation with sensitivity derivatives [36, 37, 38, 39. By using sensitivity information, it is possible to reduce the workload by one to two orders of magnitude over the standard Monte Carlo method at the cost of only one extra (tangent) linear problem to solve per parameter. We implement both the forward and tangent linear problems within DOLFIN/FEniCS [40, 41]. This work is mainly a biomechanics application of that existing method with an extension to random fields. The accelerated MC method is a Control Variates method [42] using a first order Taylor expansion as a control variable.

Most existing FE codes are not able to compute the tangent linear model and the sensitivity derivatives. However, it is possible with DOLFIN for a wide range of complex models with very little effort from the high-level Unified Form Language (UFL) description [43] of our finite element models. The Python toolbox Chaospy [44] is also employed to easily generate different stochastic objects like probability density function or global sensitivity Sobol indices. Sobol indices show which parameters have a significant influence on a given quantity of interest [45, 46].

The standard Monte Carlo method is used in this work as a commonly 
understood baseline for performance comparison. However the sensitivity derivative method could be used in conjunction with other variance reduction techniques, such as multi-level Monte Carlo methods [47]. It could also be combined with quasi-random low-discrepancy sequences, e.g. Sobol sequences [48] to improve the rate of convergence.

An outline of this paper is as follows: in section 2 the general stochastic problem setting is given. Different approaches to model uncertainty with random variables/fields are given. In section 3 the standard and the sensitivity derivative Monte Carlo methods are described before continuing to section 4 which specifies their numerical implementation in FEniCS and describes the parallel computing tools we use. In section 5 , we present a simple benchmark problem (stochastic hyperelastic beam) and all associated data are made available as supplementary material. Finally, numerical results of a stochastic analysis of brain deformation are presented and discussed in section 6 for two (isotropic and anisotropic) hyperelastic models. We propagate the assumed uncertainty in the constitutive model through a finite element model of the brain. The constitutive models are not calibrated with real data. This method could be improved if the application is checked in an experimental study. So the novelty of the work is primarily in the numerical implementation of the sensitivity derivatives Monte Carlo method and the proposed method can be used easily for other cases and material models.

\section{Stochastic modelling and problem setting}

Hyperelasticity is a widely used model to describe the behavior of soft tissues. It is possible to express the associated mechanical problem as a minimisation of the total potential energy to obtain the displacement field $u$. We want to find the solution $u$ of a non-linear system of equations such as:

$$
F(u, \omega)=0,
$$

where $\omega$ are some parameters of the system of equations. In most applications, in particular in biomechanics, where parameters can vary by several orders of magnitude, the parameters of the model are not known deterministically. We consider instead that the parameters $\omega$ of the system are uncertain. The displacement field consequently depends on the values of the parameters $\omega$. To simplify the notations, the spatial position $x$ is omitted but it is understood that $u$ also depends on $x$. 
The associated uncertainty propagation problem can then be stated as follows. Consider a probability space $\left(\Omega_{p}, \mathcal{F}, P\right)$ where $\Omega_{p}$ is the sample space, $\mathcal{F}$ is a $\sigma$-algebra of subsets of $\Omega_{p}$ and $P$ is a probability measure. Given a quantity of interest on the solution $\psi$, find the expected value of the quantity of interest:

$$
\mathbb{E}[\psi(u(\omega))]:=\int_{\Omega_{p}} \psi(u(\omega)) d P(\omega) .
$$

For example to evaluate the mean value of $u$ denoted by $\bar{u}, \psi: u \mapsto \psi(u)=u$ and to evaluate the variance of $u, \psi(u)=u^{2}-\bar{u}^{2}$. Usually, eq. (2) can not be solved analytically and numerical methods are needed to approximate numerically this integral, the most commonly used techniques belong to the family of Monte Carlo methods. The standard Monte Carlo methods solve the set of equations for the displacement field $u$ for a "sufficiently large" number $Z$ of parametric values $\left\{\omega_{z}\right\}_{z=1}^{Z}$. For each realization of the parameters $\omega_{z}$, the quantity of interest $\psi(u)$ is stored, and the collection of solutions $\left\{\psi\left(u_{z}\right)\right\}_{z=1}^{Z}$ can be statistically analyzed. Note that in our numerical examples we store a functional $\psi$ depending on the solution (i.e. $U_{x}, U_{y}$ or $U_{z}$ on a subdomain).

To solve the problem (eq. (2)) by the Monte Carlo method, it is necessary to generate a large number of realizations of $\omega$ by using classical random generators. The Monte Carlo estimator and the convergence rate of a Monte Carlo method are given in the next section.

In the case of correlated random fields 35 the generation is more complex. One method consists in generating a Gaussian random field and then considering different types of distributions through some transformation of this Gaussian field. For example, the exponential of a Gaussian field is a log-normal field. A log normal distribution is often well-suited to represent physical parameters because the probability of drawing non-physical negative-valued parameters is null. Given the correlation function and the mean value of the Gaussian field, many techniques exist to generate the field on a specific domain, e.g. Fast Fourier Transform (for stationary fields) [49], Karhunen-Loève expansion [35] or with a stochastic partial differential equation approach [34]. The Karhunen-Loève expansion is used in this work and more details are given in section 6 .

A Monte Carlo method with use of sensitivity derivatives to reduce the error is presented in section 3 in a finite element context after noting the basis of the standard one.

In our numerical simulations we will perform a sensitivity analysis to understand the impact on the outputs of the model of sources of uncer- 
tainty in its inputs. Sobol indices show which parameters have a significant influence on a given quantity of interest [45, 46. The Sobol method is a variance-based sensitivity analysis and can detect interaction effects with independent variables. The fist-order and total-effect Sobol indices are the most commonly used for a global sensitivity analysis. For example if the quantity of interest $\psi$ depends on $n$ parameters $\omega=\left(\omega_{1}, \omega_{2}, \ldots, \omega_{n}\right)$, the first-order index for the parameter $\omega_{i}$ is:

$$
\mathrm{S}_{i}=\frac{\operatorname{Var}_{\omega_{i}}\left(\mathbb{E}\left[\psi \mid \omega_{i}\right]\right)}{\operatorname{Var}(\psi)}
$$

where Var denotes the variance and $\mathbb{E}\left[\psi \mid \omega_{i}\right]$ is the expected value of $\psi$ knowing $\omega_{i}$ (the variance is hence taken over a $n$-1-dimensional parameter space). We evaluate the variance over all possible values $\omega_{i}$. The total effect index is evaluated as:

$$
\mathrm{ST}_{i}=\frac{\underbrace{\omega_{j}}_{j \neq i}\left[\operatorname{Var}(\psi \mid \underbrace{\omega_{j}}_{j \neq i})\right]}{\operatorname{Var}(\psi)},
$$

In eq. (4), we evaluate the expected value over a one-dimensional parameter space. We evaluate the expected value over all possible values $\omega_{j}$. Note

that the following property can be obtained: $0 \leq S_{i} \leq S T_{i} \leq 1$. The equality $S_{i}=S T_{i}$ means that there is no interaction between $\omega_{i}$ and the other variables.

\section{Finite element propagation of uncertainty}

\subsection{Standard Monte Carlo method}

The classical Monte Carlo estimator [24] can be used to evaluate an approximation of the expected value of the quantity of interest in eq. (2):

$$
\mathbb{E}[\psi(\omega)] \approx \mathbb{E}^{\mathrm{MC}}[\psi(\omega)]:=\frac{1}{Z} \sum_{z=1}^{Z} \psi\left(\omega_{z}\right),
$$

where $\omega_{z}$ is a realization of $\omega$ according to the distribution of the random parameters. This is a totally non-intrusive method because each evaluation $\psi\left(\omega_{z}\right)$ can be made by using an existing deterministic solver with different 
parameters $\omega_{z}$. Moreover, all the evaluations can be performed independently and in parallel without communication. The convergence of this process is very slow with a rate of convergence to $\mathcal{O}(1 / \sqrt{Z})$. However the rate of convergence is independent of the stochastic dimension which is a major advantage for problems with high-dimensional parameter spaces. Using the Central Limit Theorem [24] it can be shown that the above estimator converges in law under very weak assumptions to the exact expectation:

$$
\lim _{Z \rightarrow \infty} \frac{\left\|\mathbb{E}^{\mathrm{MC}}[\psi(\omega)]-\mathbb{E}[\psi(\omega)]\right\|}{\sqrt{\frac{\mathbb{V}[\psi(\omega)]}{Z}}}=\nu,
$$

where $\nu$ is a standard normal random variable $N(0,1)$. The above result is a probabilistic result on the error induced by the standard Monte Carlo estimator. $\mathbb{V}[\psi(\omega)]$ is the variance of the estimator $\psi(\omega)$. The number of realizations $Z$ can be very large to achieve an accurate solution. Instead of employing a classical random generator, quasi Monte Carlo methods use low discrepancy sequences, e.g. Sobol sequences [48, to improve the rate of convergence to $\mathcal{O}\left((\log Z)^{M} Z^{-1}\right)$ for some constant $M$ which itself does depend on the stochastic dimension. Alternatively to reduce the error, we can reduce the variance of the estimator, see eq. (6). This is the objective of the variance reduction method described in section 3.2 which use sensitivity derivatives. Of course, this method could be combined with a quasi-random generator to further increase the convergence but a standard Monte Carlo method, as a commonly understood baseline for performance comparison, is used in this work.

\subsection{Monte Carlo method with use of sensitivity derivatives}

As mentioned previously, to reduce the error in the Monte Carlo estimator, we can reduce the variance of the estimator. An improved variance reduction technique is introduced in [36] by coupling sensitivity derivatives to the Monte Carlo estimator. The sensitivity derivatives are the derivatives of the quantity of interest with respect to the parameters evaluated at the mean of the parameters. Here only the first derivative $D_{\omega}$ is taken into account but the method can be generalized by using higher order derivatives [37. The accelerated MC method is a Control Variates method using a first order Taylor expansion as a control variable. $D_{\omega}$ is the Fréchet derivative of the quantity of interest $\psi$ with respect to the parameter $\omega$ about the mean parameter $\bar{\omega}$. The expected value of a quantity of interest becomes:

$$
\mathbb{E}_{1}^{\mathrm{SD}-\mathrm{MC}}[\psi(\omega)]:=\frac{1}{Z} \sum_{z=1}^{Z}\left[\psi\left(\omega_{z}\right)-D_{\omega}[\psi(\bar{\omega})]\left(\omega_{z}-\bar{\omega}\right)\right] .
$$


Because of factor $\left(\omega_{z}-\bar{\omega}\right)$ in eq. (7) with $\bar{\omega}$ the mean value of $\omega$, the estimator given by the standard (MC) and the sensitivity derivatives (SD-MC) Monte Carlo approaches are similar when $Z$ tends to infinity. If the variance of SD$\mathrm{MC}$ estimator is lower than the variance of the $\mathrm{MC}$ estimator, then the error in eq. (6) is reduced. Quantitatively, the sensitivity derivative correction will improve on the standard MC method if and only if [37]:

$$
\mathbb{V}\left[T_{1}(\psi)\right] \leq 2 \operatorname{Cov}\left(\psi, T_{1}(\psi)\right),
$$

where Cov denotes the covariance operator and $T_{1}(\psi)$ the first order Taylor series expansion of $\psi$. These results tell us intuitively that the sensitivity derivative correction will only improve on the standard Monte Carlo method if the Taylor approximation to the function about the mean parameter $\bar{\omega}$ is sufficiently good. Numerical results presented in section 6 show that the number of realizations can be reduced by one to two order of magnitude by using the sensitivity derivatives Monte Carlo (SD-MC) estimator. For some very specific cases if Eq. 8 is not verified, an improved sensitivity derivative Monte Carlo method is proposed in [37. This method adds a multiplying factor in the Taylor expansion which is very simple to calculate and ensures lower error, see [37] for more details. For the numerical simulations presented in this paper, this coefficient is close to one and is not taken into account in the MC estimator eq. (7).

\section{Numerical implementation}

\subsection{FE software}

The FEniCS Project is a collection of free software with an extensive list of features for automated, efficient solution of differential equations [50]. It is possible to derive the forward and the sensitivity derivative model using the automatic differentiation features in the Unified Form Language (UFL) 43] of the FEniCS Project [41]. For a full review of techniques that can be used to derive possibly time-dependent tangent linear and adjoint models using the DOLFIN automated finite element solver, see dolfin-adjoint [43]. For a generalized Burgers equation with stochastic viscosity, a complete Python code is given in [38. All the numerical results shown in section 6 are computed with DOLFIN. The original motivation for using DOLFIN is that it is possible to automatically derive the tangent linear models (see section 4.2) and then use it to be able to calculate the sensitivity derivatives. Furthermore, complex models can be implemented relatively straightforwardly such as non-linear anisotropic hyperelastic models [51] used in the numerical simulations in section 6 . 


\subsection{Automatically deriving tangent linear models}

In the case that the solution $u$ is discretized using finite elements, leading to a discrete space with size $Q$ and the random parameter $\omega$ is a scalar, $\omega \in \mathbb{R}$, the solution Jacobian $\frac{\mathrm{d} u}{\mathrm{~d} \omega}:=u_{\omega}$ will be a vector of size $Q$, the linearized solution operator about the solution $u, \frac{\partial F(u, \omega)}{\partial u}:=A_{u}$ will be a matrix size $Q \times Q$, and the right-hand side source term $\frac{\partial F(u, \omega)}{\partial \omega}:=f_{\omega}$ will be a vector of size $Q$. Having solved the non-linear problem eq. (1) at the mean parameter $\bar{\omega}$ to find $u(\bar{\omega})$ the following linear system at $u(\bar{\omega})$ can be solved:

$$
A_{u} u_{\omega}=-f_{\omega},
$$

to directly obtain the required derivative information $u_{\omega}=D_{\omega}[u(\bar{\omega})]$ for the sensitivity-derivative driven Monte Carlo method. Once the finite element residual equation is defined in variational form with FEniCS using UFL, the corresponding variational forms for the tangent linear model can be derived with only two function calls that invoke the automatic differentiation techniques implemented in UFL. We could try a very straightforward approach by finite differencing to evaluate directly the term $\frac{\partial F(u, \omega)}{\partial \omega}$ but in practice this approach has several limitations. First the finite difference approximation requires one functional evaluation for each degree of freedom in the solution space which is very time consuming in the context of PDEs where each evaluation necessitates to solve a system. In addition, the accuracy of the approximation is very sensitive to the spacing parameter commonly denoted by $h$. If $h$ is too large the error of approximation will be significant while a very small $h$ can be faced with numerical precision errors.

\subsection{Parallel computing}

The ipyparallel parallel computing toolbox [52] is used to distribute forward model evaluations across an 8 socket Intel Xeon E7-8880-based machine with 15 cores per socket, giving 120 cores in total. This machine is installed in the Gaia cluster at the University of Luxembourg. The message passing interface (MPI) backend is used for communication between ipyparallel engines and the ZeroMQ distributed messaging system to communicate between workers and the controller. Each ipyparallel engine uses its own MPI communicator (SELF) to solve each realization of the forward problem.

\section{Practical example (hyperelastic beam with random parameter)}

Before continuing to the stochastic finite element analysis of brain deformation, a simple practical example is given in this section. The complete 
code for this example, developed within the FEniCS open-source software environment is made available in [53. This includes a Docker image containing the complete software environment for execution. Through this example, the user can become quickly familiar with the FEniCS environment and then adapt and change the code for his own needs. For example, the constitutive law and the distribution of the random parameters can be altered within only a few lines of Python code.

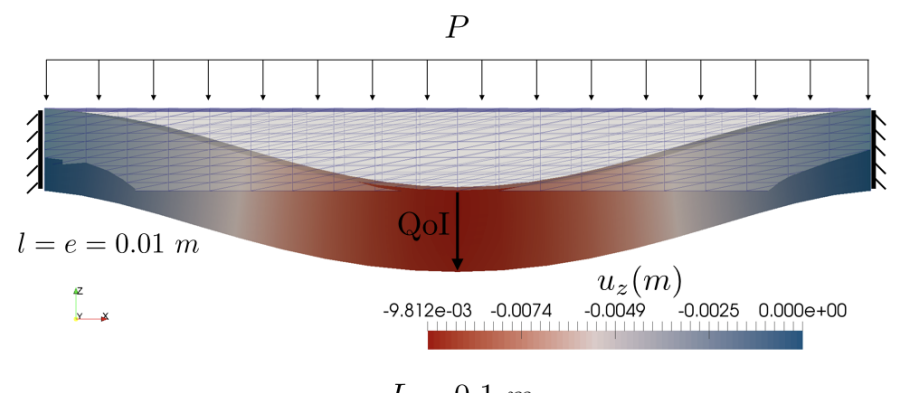

$$
L=0.1 m
$$

Figure 1: Hyperelastic beam deformation (mesh in the initial configuration and deformed configuration for one Monte Carlo realisation).

The equations to describe the behavior of hyperelastic solids are recalled in section 6. The objective in this practical example is to determine the expected value of the displacement of the center of the beam (coordinates $(L / 2,0,0))$ attached at its ends and with loading pressure $P=50 \mathrm{~Pa}$ (see Fig. 1). A Neo-Hookean solid is considered with stochastic Young's modulus modeled by:

$$
E(\omega)=E_{0}(1+2 \omega)
$$

with $\omega$ a beta $(2,2)$ random variable and $E_{0}=10^{4} \mathrm{~Pa}$. Monte Carlo results for $Z=50$ realizations of Young's modulus with and without sensitivity derivatives are plotted in Fig. 2. The variance in the results is smaller when sensitivity derivatives are taken into account in the Monte Carlo estimator. The Monte Carlo method with sensitivity derivatives will eventually converge faster to the expected value of the quantity of interest (QoI).

\section{Stochastic finite element analysis of brain deformation}

In this section we present the results of a stochastic finite element analysis of brain deformation. Two constitutive models with uncertain parameters are considered: a Mooney-Rivlin (MR) material [54] and a Holzapfel 


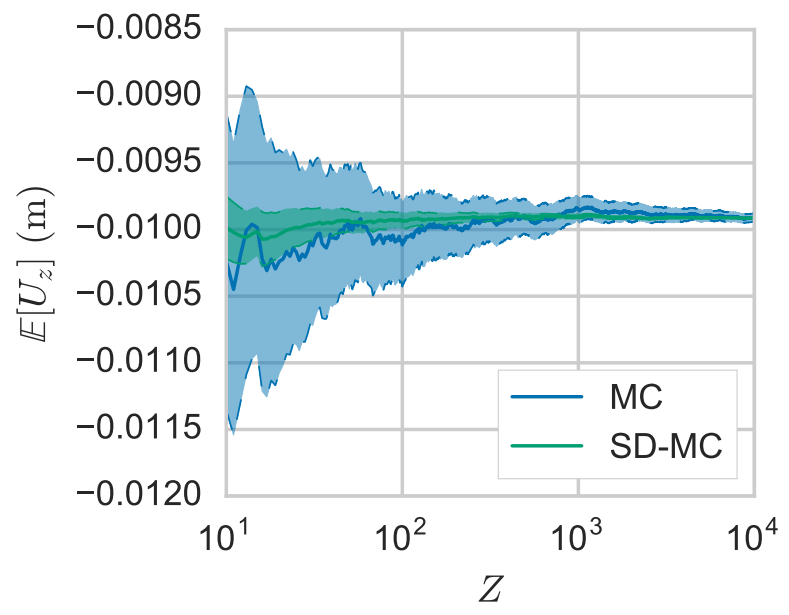

Figure 2: Evolution of the estimator $\mathbb{E}\left[U_{z}\right]$ for the practical example (beam), estimator of the mean value of the displacement of the center of the beam in the $z$ direction as a function of the number of realizations $Z$. $Z=50$ Monte Carlo realizations of Young's modulus are performed with and without sensitivity derivatives. The variance in the results is smaller when sensitivity derivatives are taken into account in the Monte Carlo estimator. The blue and red shadings represent the confidence intervals for the two methods with a confidence level at $95 \%$. 
and Ogden (HO) model [51, 55]. These simulations aim at illustrating the uncertainty propagation methods we developed in the general framework of soft-tissue undergoing finite deformations.

\subsection{Geometry and boundary conditions}

The boundary conditions we used in the simulations are similar to those used in [54] where the authors give a modeling of brain shift phenomenon for different craniotomies and solid models. A part of the brain is fixed to limit the movement of the brain and a pressure variation fixed at $10 \mathrm{mmHg}$ $(1333 \mathrm{~Pa})$ in the area of operation. The mesh is composed of 30805 elements and 6148 nodes. A schematic description of the chosen boundary conditions are provided in Figure 3 .
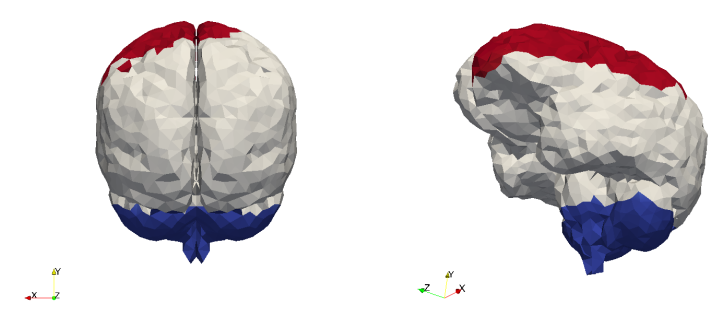

Figure 3: Schematic description of the chosen boundary conditions. A part of the brain is fixed (blue zone) and a normal pressure variation is imposed at $10 \mathrm{mmHg}(1333 \mathrm{~Pa})$ in the area of operation (red zone).

\subsection{Governing equations}

For a domain $\Omega_{0}$ with smooth boundary $\partial \Omega_{0}$ and $x \in \Omega_{0}$ the spatial position, the hyperelastic equations consists in finding the displacement $\mathbf{u}^{*}$ among all displacement fields $\mathbf{u}$ which minimize the total potential energy while satisfying the boundary conditions. The strain energy density function is denoted by $\mathcal{W}(\mathbf{u})$ and $\mathbf{f}$ refers to external loading (traction force, body force, etc.). The minimisation problem writes:

$$
\mathbf{u}^{*}=\underset{\mathbf{u} \in\left[H_{D}^{1}\left(\Omega_{0}\right)\right]^{3}}{\arg \min }\left\{\int_{\Omega_{0}} \mathcal{W}(\mathbf{u}) \mathrm{d} x_{0}-\langle\mathbf{f}, \mathbf{u}\rangle\right\}=L(\mathbf{u}),
$$

where $\left[H_{D}^{1}\left(\Omega_{0}\right)\right]^{3}$ is the usual vector-valued Sobolev space of square integrable functions with square integrable derivatives that satisfies the given 
Dirichlet boundary conditions and $d x_{0}$ is a measure on $\Omega$. The directional Fréchet derivative of $L$ vanishes at the minimum points:

$$
\forall \tilde{\mathbf{u}} \in H_{0}^{1}\left(\Omega_{0}\right): \quad F\left(\mathbf{u}^{*} ; \tilde{\mathbf{u}}\right)=D_{\tilde{u}} L\left(\mathbf{u}^{*}\right)=0 .
$$

In the presence of random coefficients, $F$ also depends on random parameters $\omega$ and an equation of the same type as ?? is obtained. To solve the non-linear problem a Newton method with a third-order backtracking linesearch from SNES [56] was used. The symbolic differentiation capabilities of UFL are used to derive the Jacobian of the forward model for the Newton solver. The linear systems arising from the Newton iterations are solved using a conjugate gradient method preconditioned using algebraic multigrid (Hypre BoomerAMG [57]) interfaced from PETSc [56].

\subsection{Constitutive equations}

We choose two hyperelastic models. To describe the kinematics, usual operators and notations are used: the deformation gradient $\mathrm{F}=\mathrm{I}+\nabla \mathbf{u}$ and the right Cauchy-Green tensor C associated with F:

$$
\mathrm{C}:=\mathrm{F}^{T} \mathrm{~F}
$$

For the Mooney-Rivlin (MR) material, the strain energy density function depends on three parameters $\left\{C_{1}, C_{2}, D_{1}\right\}$ :

$$
\mathcal{W}_{\mathrm{MR}}=C_{1}\left(\bar{I}_{1}-3\right)+C_{2}\left(\bar{I}_{2}-3\right)+D_{1}\left(I_{3}^{1 / 2}-1\right)^{2} .
$$

The modified first and second invariants $\bar{I}_{1}$ and $\bar{I}_{2}$ are defined by:

$$
\bar{I}_{1}:=J^{-2 / 3} I_{1} \text { and } \bar{I}_{2}:=J^{-4 / 3} I_{2},
$$

where $I_{1}, I_{2}$ and $I_{3}$ are the unmodified invariants of $C$ given by:

$$
\begin{aligned}
I_{1}(\mathrm{C}) & :=\operatorname{tr}(\mathrm{C}), \\
I_{2}(\mathrm{C}) & :=\frac{1}{2}\left((\operatorname{tr} \mathrm{C})^{2}-\left(\operatorname{tr}^{2}\right)\right), \\
I_{3}(\mathrm{C}) & :=\operatorname{det}(\mathrm{F})^{2}=J^{2} .
\end{aligned}
$$

Alternatively the Holzapfel and Ogden HO [51, 55] anisotropic model, the strain energy density function is expressed as:

$$
\mathcal{W}_{\mathrm{HO}}=\mathcal{W}_{\text {iso }}(\mathrm{F})+\mathcal{W}_{\text {vol }}(J)
$$


$\mathcal{W}_{\text {iso }}$ and $\mathcal{W}_{\text {vol }}$ are the isochoric and volumetric part of the strain energy density function respectively. The volumetric part can be evaluated as a function of the bulk modulus $\kappa$ of the material and $J$ :

$$
\mathcal{W}_{\mathrm{vol}}=\frac{\kappa}{4}\left(J^{2}-1-2 \cdot \ln J\right) \text {. }
$$

The isochoric part takes the following form:

$$
\begin{array}{r}
\mathcal{W}_{\text {iso }}=\frac{a}{2 b} \exp \left[b\left(\mathrm{I}_{1}-3\right)\right]+\sum_{i=f, s} \frac{a_{i}}{2 b_{i}} \exp \left[b_{i}\left(\mathrm{I}_{4 i}-1\right)^{2}\right] \\
+\frac{a_{\mathrm{fs}}}{2 b_{\mathrm{fs}}}\left(\exp \left[b_{\mathrm{fs}} \mathrm{I}_{8 \mathrm{fs}}^{2}\right]-1\right),
\end{array}
$$

with:

$$
\mathrm{I}_{4 f}=\boldsymbol{f}_{0} \cdot \mathrm{C} \cdot \boldsymbol{f}_{0}, \mathrm{I}_{4 s}=\boldsymbol{s}_{0} \cdot \mathrm{C} \cdot \boldsymbol{s}_{0} \text { and } \mathrm{I}_{8 \mathrm{fs}}=\boldsymbol{f}_{0} \cdot \mathrm{C} \cdot \boldsymbol{s}_{0} .
$$

The transversely isotropic behavior can be obtained by not taking account at all in the model the parameter $a_{\mathrm{fs}}, b_{\mathrm{fs}}, a_{s}$ and $b_{s}$, while the isotropic one corresponds to remove also in addition the two parameters $a_{f}$ and $b_{f}$. This kind of model is frequently used to model orthotropic materials (e.g. muscle with fibers). The vectors $\boldsymbol{f}_{0}, \boldsymbol{s}_{0}$ (and $\boldsymbol{n}_{0}$ ) are the unit base vectors normal to the planes of symmetry. For the numerical simulations, these models are implemented within DOLFIN/FEniCS and the method of manufactured solutions [58] is employed to verify the implementation.

\subsection{Stochastic modelling}

We choose the material properties according to the wide variety of experimental data provided in the literature, e.g. [20, 54, 59, 16]. Few data are available to our knowledge for the anisotropic Holzapfel-Ogden model [51, 55] but there are recent articles that have focused on the anisotropic modelling of the brain tissue such as [21, 60]. We do not calibrate the probability distributions used for each parameter using atlases but generate these distributions by inspection of the literature. The purpose of the remainder of the paper is to demonstrate that given suitable statistical distributions, we can reliably and with reasonable computational costs, propagate uncertainties through the mathematical model representing the organ's behavior.

All eight parameters involved in the HO hyperelastic model are modelled with random variables with a log-normal distribution to have zero probability of drawing non-physical negative-valued parameters (see table 1). The bulk modulus is taken constant and equal to $10^{5} \mathrm{~Pa}$. The probability density function of two of them is plotted in fig. 4 . 


\begin{tabular}{rrrrrrrrr}
\hline Parameters: & $a$ & $b$ & $a_{f}$ & $b_{f}$ & $a_{s}$ & $b_{s}$ & $a_{\mathrm{fs}}$ & $b_{\mathrm{fs}}$ \\
\hline Mean (Pa) & 1180.0 & 8.0 & $18.5 \cdot 10^{4}$ & 16.0 & $2.5 \cdot 10^{4}$ & 11.1 & 2160.0 & 11.4 \\
Std (Pa) & 236.0 & 1.6 & $3.7 \cdot 10^{4}$ & 3.2 & $5.0 \cdot 10^{3}$ & 2.2 & 432.0 & 2.3
\end{tabular}

Table 1: Random parameters involved in the Holzapfel and Ogden hyperelastic model. All the parameters are modelled by random variables with a log-normal distribution. The mean value and the standard deviation are indicated.

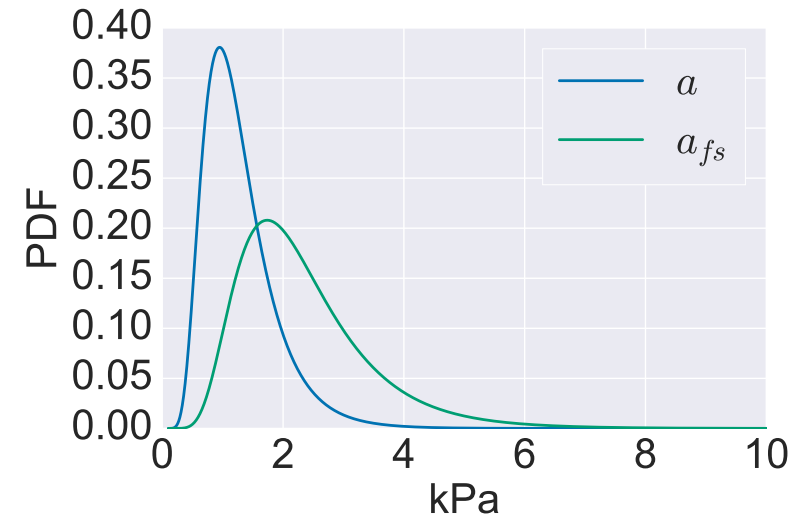

Figure 4: Probability density function of the parameters $a$ and $a_{\mathrm{fs}}$. 
The vectors $\boldsymbol{f}_{0}, \boldsymbol{s}_{0}$ involved in the HO model are chosen arbitrarily to $[0,1 / \sqrt{2}, 1 / \sqrt{2}]$ and $[0,1 / \sqrt{2},-1 / \sqrt{2}]$ respectively.

For the Mooney-Rivlin case, the uncertainty of the three parameters is taken into account by using random fields with a log-normal distribution and a correlation length equal to $5 \mathrm{~cm}$. Without available experimental data, this value has been fixed arbitrary with a reasonable order of magnitude compared to the size of the domain. As mentioned in the introduction, this work could be improved if we could have access to real data to identify and calibrate the parameters involved in the model. The mean value and the standard deviation on these random fields are indicated in table 2 . As mentioned previously, a transformation of a Gaussian fields is used to generate the target distribution, more details can be found in [61]. For example, a log-normal field is obtained by taking the exponential of a Gaussian field and if $\mu_{l}$ and $\sigma_{l}^{2}$ are the mean value and the variance of the log-normal field, the following relations give the corresponding values $\mu_{g}$ and $\sigma_{g}^{2}$ to impose at the Gaussian field:

$$
\mu_{g}=\ln \left(\mu_{l}\right)-\frac{1}{2} \ln \left(1+\sigma_{l}^{2} / \mu_{l}^{2}\right) \text { and } \sigma_{g}^{2}=\sqrt{\ln \left(1+\sigma_{l}^{2} / \mu_{l}^{2}\right)} .
$$

The Karhunen-Loève expansion of a Gaussian field $\Gamma$ is computed by solving

\begin{tabular}{rrrr}
\hline Parameters: & $C_{1}$ & $C_{2}$ & $D_{1}$ \\
\hline Mean $(\mathrm{Pa})$ & 3922.2 & 30.0 & $5.0 \cdot 10^{4}$ \\
Std $(\mathrm{Pa})$ & 392.2 & 15.0 & $2.5 \cdot 10^{4}$
\end{tabular}

Table 2: Mooney-Rivlin parameters (mean value and standard deviation of the random fields with log-normal distribution).

an eigenvalue $\left(\lambda_{i}\right.$ and $\left.\phi_{i}(x)\right)$ problem [35], this allows to obtain the following decomposition:

$$
\Gamma(x, \omega)=\Gamma_{0}(x)+\sum_{i=1}^{+\infty} \sqrt{\lambda_{i}} \phi_{i}(x) \omega_{i},
$$

with $\Gamma_{0}(x)$ the mean value of the Gaussian field. Note that in the case of a Gaussian field, each $\omega_{i}$ are independent and uncorrelated Gaussian variable with zero mean and unit variance. A random generator can then be used easily to generate different realizations. Routinely, the eigenvalues/eigenvectors system is solved for a Gaussian field with zero mean and unit variance and then this decomposition can be used for all Gaussian fields by adding the 
mean value and multiplying all the eigenvectors by the desired standard deviation. For the mesh used for the numerical simulations, the normalized eigenvalues spectrum of the Gaussian field used to generate the log-normal field is represented in fig. 5. For the sake of example, two realizations of

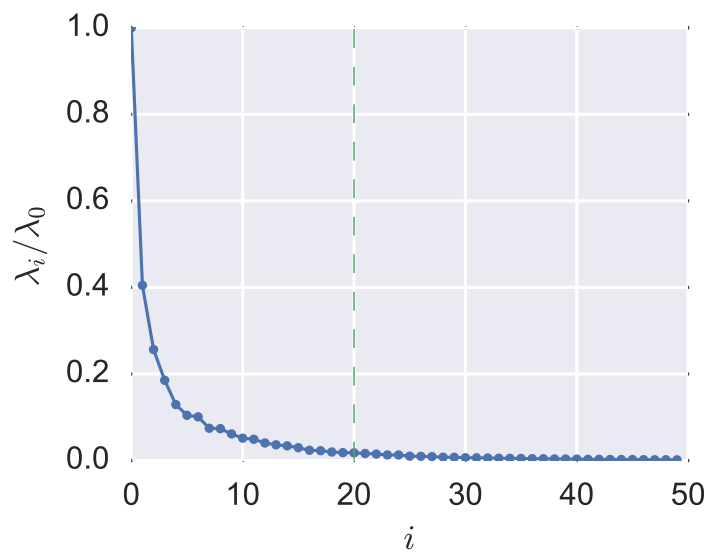

Figure 5: Karhunen-Loève expansion: normalized eigenvalues spectrum of the Gaussian field. With a transformation of a Gaussian field it is then possible to generate fields with log-normal distribution and used them to model parameter uncertainty in the MooneyRivlin hyperelastic problem. The green dashed line shows the truncation of the KL expansion at 20 modes.

the parameter $C_{1}$ are illustrated in fig. 6. The sum in eq. (22) is truncated in order to capture the majority of the energy (area under the curve in fig. 5). For the numerical simulations 20 modes are taken into account in the Karhunen-Loève expansion.

\subsection{Numerical results}

For both hyperelastic models, the displacement of a target localized in the brain is studied. The mesh used for the simulations is shown in fig. 7 in the initial and deformed configuration and contains around 20,000 degrees of freedom. The objective is to study the stochastic response (displacement) of the target given the random coefficients involved in the definition of the two hyperelastic problems. The computational time is significant and is dominated by the number of forward deterministic systems to solve in order to obtain an accurate solution to the stochastic problem. 


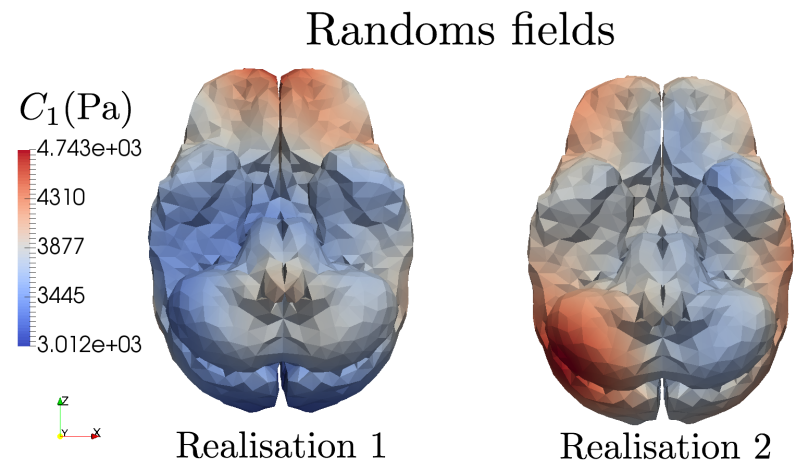

Figure 6: Random fields with log-normal distribution, two different realizations of the parameter $C_{1}$.

By using the Monte Carlo method with sensitivity derivatives (SD-MC) it is shown that the number of realizations can be reduced by one to two order of magnitude compared with a simple Monte Carlo approach. As stated in section 3.2 , for the cost of only $m$ linear systems with $m$ the stochastic dimension, the SD-MC method can be very effective. The time spent to solve these linear systems is negligible compared to the time necessary to solve all the repeated non-linear hyperelastic problems.

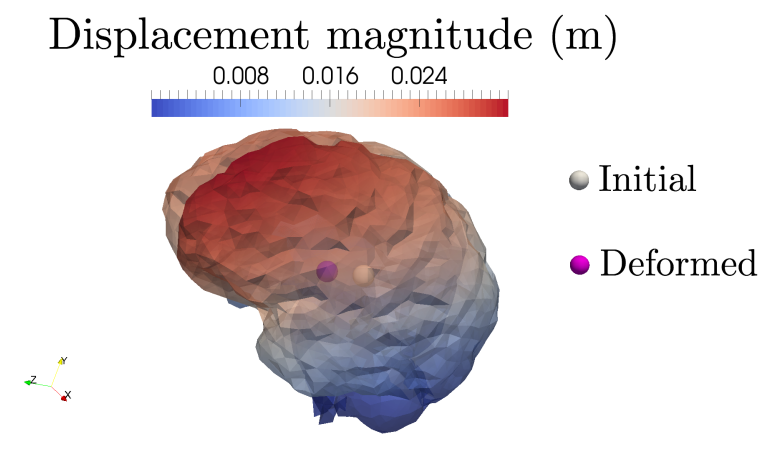

Figure 7: Mesh with around 20,000 degrees of freedom (initial configuration) and deformed mesh for one Monte Carlo realisation obtained with the HO model. The target volume is shown with a sphere. The displacement and the deformation of the target depend on the parameters. 


\subsubsection{HO model}

In fig. 8, the evolution of the estimator $\mathbb{E}\left[U_{x}\right](\mathrm{m})$ which is the expected value of the displacement of the sphere in the $x$ direction as a function of the number of realizations $Z$ is plotted. For the MC and the SD-MC methods, 12, 000 realizations are performed. The two evolutions eventually converge to the same value and incontestably the use of sensitivity derivatives clearly provides for an improved convergence behavior (few realizations for the SDMC method instead of $Z \approx 500$ for the MC estimator, see fig. 8). Of course these values depend on the quantity of interest and on the draw of sampling. There is no analytical solution to compare the results against more quantitatively but this trend is found in many other examples treated in [38, 39]. With $Z=12,000$ Monte Carlo samples, it is possible to construct

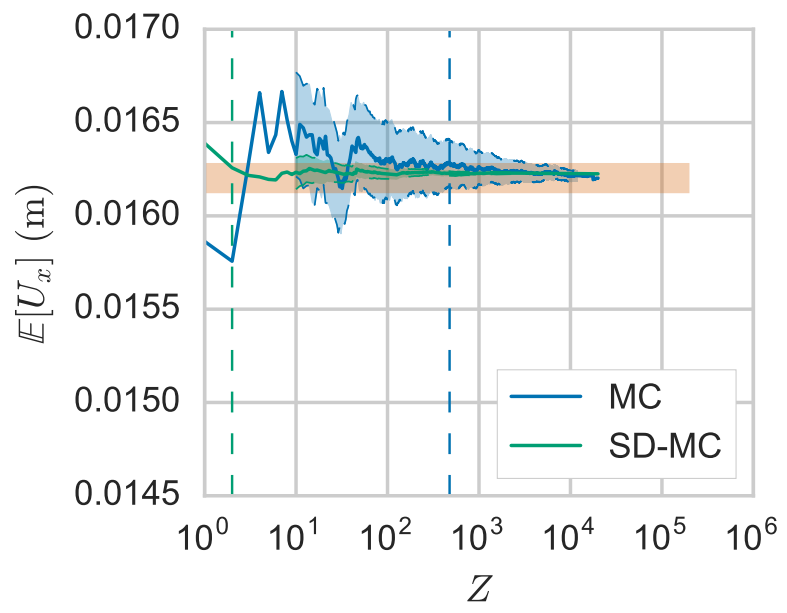

Figure 8: Evolution of the estimator $\mathbb{E}\left[U_{x}\right]$ (HO model), estimator of the mean value for the displacement in the $x$ direction of the sphere as a function of the number of realizations $Z$. Comparison between the MC and the SD-MC methods. The orange shaded box represents the region where the value of the estimator is ranged between the final estimate predicted by the MC estimator $\pm 0.5 \%$. The vertical dashed lines give the value of $\mathrm{Z}$ for each method where the curve stabilizes in this region. The SD-MC estimator converges faster than the classical MC estimator. The blue and red shadings represent the confidence intervals for the two methods with a confidence level at $95 \%$.

a confidence interval on the position of the target. This confidence interval at a $95 \%$ confidence level is plotted in fig. 9 as well as the displacement and the deformation of the target (sphere) for two different realisations of 
random parameters. The global sensitivities using the polynomial chaos

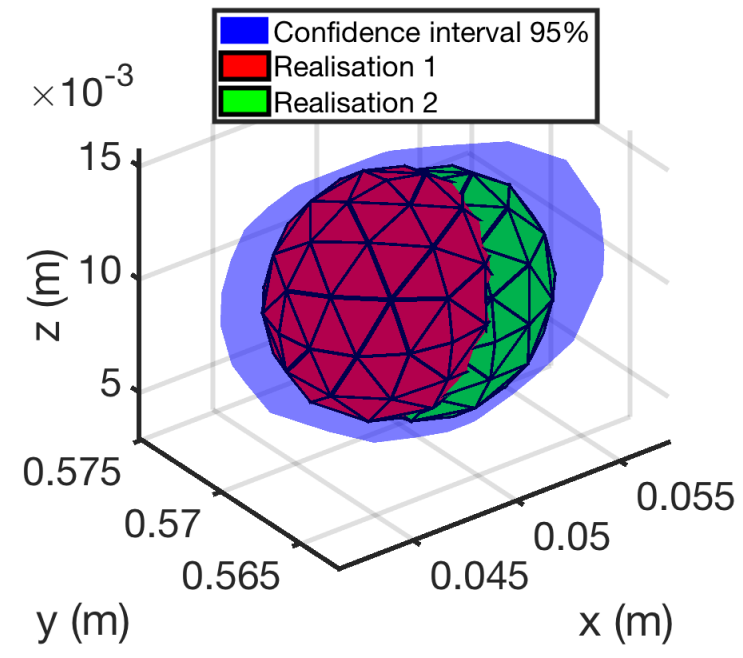

Figure 9: Displacement and deformation of the sphere (HO model): confidence interval at a $95 \%$ confidence level.

expansion output of the MC method is calculated [62] using Sobol global sensitivity indices, see [48, 45] for more details. In the polynomial chaos expansion approach we expand the stochastic solution $u(x, \omega)$ with $\omega \in \mathbb{R}^{M}$ in a basis $H_{\alpha}(\omega)$ of polynomials of dimension $M$ and order $p$ [63, 64]:

$$
u(x, \omega)=\sum_{\alpha \in \mathcal{J}_{M, p}} c_{\alpha}(x) H_{\alpha}(\omega),
$$

with $\alpha \in \mathcal{J}_{M, p} . \mathcal{J}_{M, p}$ is the set of multi-indices given by:

$$
\mathcal{J}_{M, p}=\left\{\alpha \in \mathbb{N}_{0}^{(\mathbb{N})}\left|\alpha=\left\{\alpha_{1}, \ldots, \alpha_{j}, \ldots, \alpha_{M}\right\}, \alpha_{j} \in \mathbb{N}_{0},\right| \alpha \mid=\sum_{j=1}^{M} \alpha_{j} \leq p\right\} .
$$

The dimension $N$ of $\mathcal{J}_{M, p}$ increases rapidly with $M$ and $p$ :

$$
N=\operatorname{dim}\left(\mathcal{J}_{M, p}\right)=(M+p) ! /(M ! p !),
$$

making this technique suitable for problems of low to moderate stochastic dimension [26]. Different methods exist to calculate the coefficients $c_{\alpha}(x)$ of the polynomial chaos expansion. We choose to solve for the coefficients using a collocation approach and the solution of a least-squares minimisation 
problem as in 39]. The Sobol global sensitivity indices characterize how the uncertainty of the inputs influence the outputs. For two quantities of interest, the Sobol global sensitivity indices are given. We have used a polynomial chaos expansion of order 3 and dimension 8 to calculate the Sobol global sensitivity indices for the numerical simulations of the $\mathrm{HO}$ model. The total sensitivity index is nearly equal to the first order sensitivity index for all variables and all quantities of interest fig. 10. This means that there is little interaction between the random variables. The results show that only parameters $a$ and $b$ affect significantly the norm of the displacement of the sphere and that $a_{s}$ has only a slight influence. On the other hand, if the quantity of interest is only the displacement in the $\mathrm{z}$ direction, then more parameters become influential, in particular $a$ and $a_{\mathrm{fs}}$.

These results highlight the importance of the choice of the parameters depending on the quantity of interest to the user and confirm that some parameters may not be activated depending on the boundary conditions and quantities of interest.

\subsubsection{Mooney-Rivlin model}

When the stochastic dimension is large, it is more suitable to turn to sampling methods to calculate the global sensitivity indices instead of polynomial chaos expansion techniques which were used previously. The Saltelli's sampling scheme allows to reduce the error rates in the resulting sensitivity index calculation [46]. The workload is significant to obtain accurate Sobol indices. In order to reduce the computational time, only the total order sensitivity indices are computed with 12, 200 realizations and a $95 \%$ confidence interval is indicated. For one quantity of interest (displacement magnitude of the sphere), these indices are plotted in fig. 11. It is recalled that 20 modes are used in the Karhunen-Loève expansion to generate the three random coefficients of the Mooney-Rivlin model. The total stochastic dimension is therefore equal to 60 because we have three parameters $C_{1}$, $C_{2}$ and $D_{1}$. Obviously, for the quantity of interest studied (displacement magnitude of the sphere), from fig. 11, the global sensitivity analysis shows that only few modes play an important role in the variance of the quantity of interest. Clearly, the uncertainty on $C_{2}$ does not affect the variance of the displacement of the sphere, see fig. 11 and only five (maximum 10) modes could be kept for $C_{1}$ and $D_{1}$ to obtain almost the same results. The estimators of the standard deviation of the displacement of the sphere in the $x$ direction as a function of the number of realizations are plotted in fig. 12(b). The orange shaded box represents the region where the value of the estimator ranges between the final estimate predicted by the MC estimator $\pm 2 \%$. 


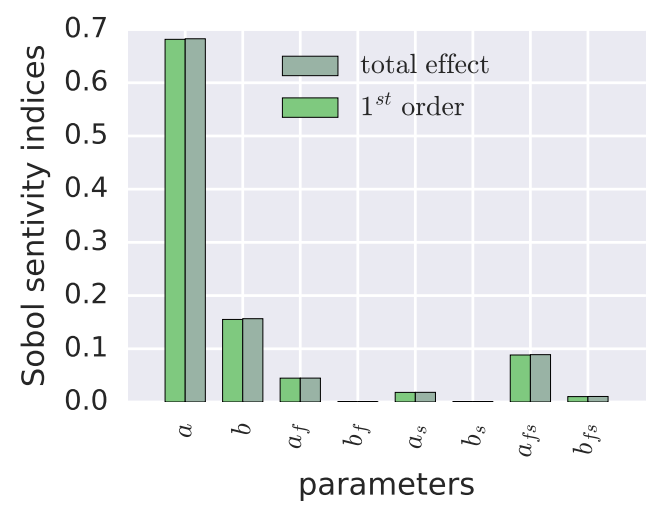

(a) Quantity of interest: displacement magnitude.

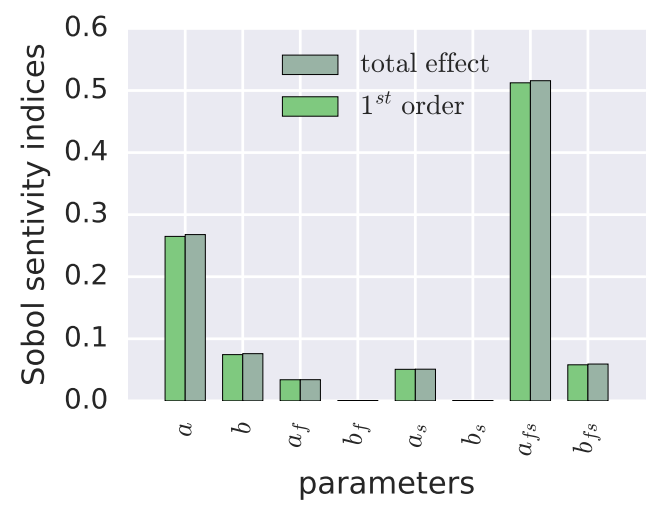

(b) Quantity of interest: displacement in the $\mathrm{z}$ direction.

Figure 10: Global sensitivity analysis (HO model). 


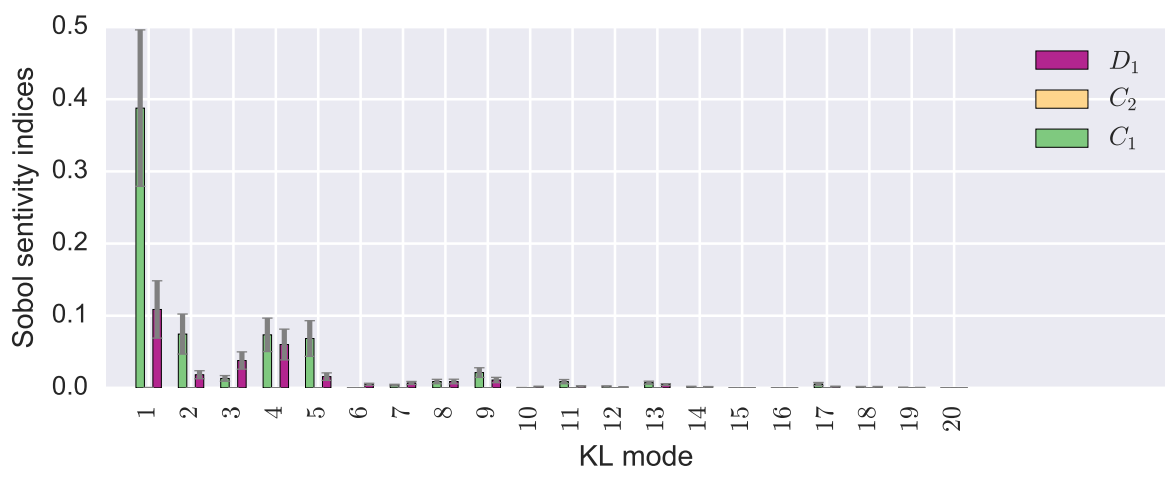

Figure 11: Global sensitivity analysis (Mooney-Rivlin material). The Sobol indices are computed with 12,200 realisations with the Saltelli's sampling scheme. A confidence interval at a $95 \%$ confidence level is indicated. Note that $C_{2}$ bars are almost zero and not visible.

The vertical dashed lines give the value of $\mathrm{Z}$ for each method where the curve stabilizes in this region. With use of sensitivity derivatives (SD-MC) the benefits are significant. The SD-MC estimator of the standard deviation settles down very rapidly to the converged answer, requiring only around 500 samples versus around 4500 samples for the standard Monte Carlo method, see fig. 12(b), The evolution of the estimators for the mean displacement of the sphere in the $\mathrm{x}$ direction $(2.9 \mathrm{~cm})$ are shown in fig. $12(\mathrm{a})$, The trend of superior performance of the $\mathrm{SD}-\mathrm{MC}$ over the MC estimator, $Z \approx 30$ versus $Z \approx 1000$ to achieve convergence, is repeated. In our numerical results, we also plot for the different estimators the confidence intervals with a confidence level at $95 \%$. These confidence bounds have been evaluated by the Strong Law of Large Numbers. For $Z$ large,

$$
\frac{\mathbb{E}[\psi]-\mathbb{E}^{\mathrm{MC}}[\psi]}{\sqrt{\operatorname{Var}^{\mathrm{MC}}[\psi]}},
$$

is approximately distributed as a standard normal distribution $\mathrm{N}(0,1)$. For the confidence interval of the variance only in the case of the standard Monte Carlo approach, we have used a different well known statistical formula involving the Chi-squared distribution with $Z$ degrees of freedom noted $v$. 
The confidence interval for the variance with safety factor $1-\alpha$ is therefore:

$$
\left[\frac{(Z-1) \operatorname{Var}^{\mathrm{MC}}[\psi]}{v_{1-\alpha / 2}}, \frac{(Z-1) \operatorname{Var}^{\mathrm{MC}}[\psi]}{v_{\alpha / 2}}\right],
$$

where $v_{\alpha / 2}$ and $v_{1-\alpha / 2}$ are the quantiles of order $\alpha / 2$ an $1-\alpha / 2$ for the Chi-squared distribution.

\section{Conclusion and future work}

We presented a general approach for the stochastic finite element analysis of soft tissue deformation. We applied the approach to a simple academic example and to the stochastic deformation of a brain. We considered two different hyperelastic models. Random variables/fields were used to account for uncertainties in material properties and advanced Monte Carlo methods were employed to provide statistical solutions and global sensitivity analyses. By using sensitivity derivatives, we showed that the error of the Monte Carlo process can be improved significantly compared to the classical approach, thus reducing computational expenses by several orders of magnitude to obtain an accurate solution. We expressed the finite element models in variational form using the high-level Unified Form Language (UFL) and made the code and data available online for the practical example. The advantages of such a methodology are:

- The implementation of complex hyperelastic models is simple and the material model can be modified by altering only a few lines of code;

- The ability to derive the tangent linear model automatically and use it to efficiently calculate the required derivative information;

- The repeated simulation of the forward problem is performed in parallel across a cluster using the ipyparallel and mpi4py software tools to save on computational time.

In spite of the progress shown in this paper, a lot of work remains to be done to better understand the role of uncertainties in biomechanics:

Uncertainties in geometry and boundary conditions. In most (bio)mechanics problems, the solution is strongly sensitive to boundary conditions and geometry. We did not account for this sensitivity here. Avenues to investigate this issue include using parametric descriptions of the geometry using nonuniform rational B-splines (NURBS) or level sets. 


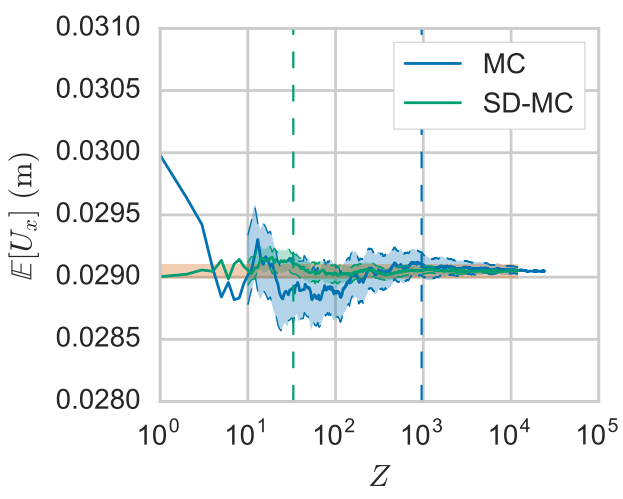

(a) Estimator of the mean value of $U_{x}$.

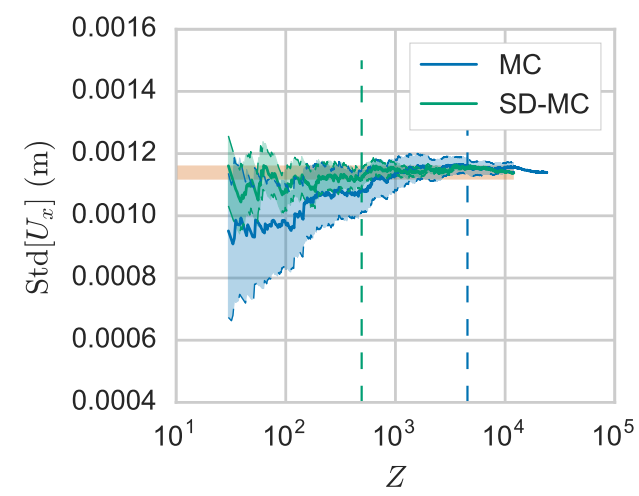

(b) Estimator of the standard deviation of $U_{x}$.

Figure 12: MR model: evolution of the two estimators $\mathbb{E}\left[U_{x}\right]$ and $\operatorname{Std}\left[U_{x}\right]$ (expected value and standard deviation) as a function of the number of realizations $Z$. $U_{x}$ is the displacement in the $x$ direction of the sphere. Comparison between the MC and the SD-MC methods. The orange shaded box represents the region where the value of the estimator is ranged between the final estimate predicted by the MC estimator $\pm 2 \%$. The vertical dashed lines give the value of $\mathrm{Z}$ for each method where the curve stabilizes in this region. The SD-MC estimator converges faster for the expected value and for the standard deviation of $\left[U_{x}\right]$. The blue and red shadings represent the confidence intervals for the two methods with a confidence level at $95 \%$. Note that the confidence interval for the standard deviation for the Monte Carlo method is large and not symmetric about the point estimate. 
Model and discretization error. Being able to identify the relative importance of parameters is useful information to the clinician. However, additional insights could be provided by developing tools able to help the user select the best model given experimental data and a quantity she/he is interested in. Doing this requires solving (stochastic) inverse problems and would also enable the stochastic identification of material parameters at a given point within an organ with only sparse measurements. In turn, by providing a spatial variation of sensitivities of the material parameters to data, such stochastic inverse approaches enable the optimization of sensor placement to decrease uncertainty on the evaluation of parameters of interest and on the selection of the most suitable model.

Confrontation to experimental data. We assumed in this paper that the probability distribution of all parameters was known a priori, but we did not build it explicitly from databases or atlases. It would be necessary to build a pipeline able to construct the required statistical information for the parameters of various models and enrich it as new information is being acquired. In particular, because of the large variability in tissue models, it would be desirable to enrich prior information provided by atlases by data acquired on a specific patient. This could be done using Bayesian inference [65].

Acceleration. In spite of being much faster than the Monte Carlo method, the algorithms we developed here are still too slow to be used in clinical practice. To overcome this difficulty, we are developing adaptive procedures and surrogate modelling approaches to accelerate the investigation of the parameter space.

\section{Supplementary material}

Complete code to produce the results in section 5 is given in 553 .

\section{Acknowledgements}

We thank the financial support of the European Research Council Starting Independent Research Grant (ERC Stg grant agreement No. 279578) entitled 'Towards real time multiscale simulation of cutting in non-linear materials with applications to surgical simulation and computer guided surgery.' Jack S. Hale is supported by the National Research Fund, Luxembourg, and cofunded under the Marie Curie Actions of the European Commission 
(FP7-COFUND) Grant No. 6693582. The experiments presented in this paper were carried out using the HPC facilities of the University of Luxembourg. We also thank the funding from the Luxembourg National Research Fund (INTER/MOBILITY/14/8813215/CBM/Bordas) and the internal MOMENTUM project at the University of Luxembourg. We thank Professor Ron Kikinis, Professor Karol Miller, Professor Adam Wittek, Professor Michel Audette and Dr Angela Mihai for helpful discussions on the mechanics of the brain and on clinical workflows.

\section{References}

[1] I. Babuška, C. Baumann, J. Oden, A discontinuous hp finite element method for diffusion problems: 1-d analysis, Computers \& Mathematics with Applications 37 (9) (1999) 103-122. doi:http://dx.doi.org/10. 1016/S0898-1221(99)00117-0.

[2] S. Bordas, M. Duflot, Derivative recovery and a posteriori error estimate for extended finite elements, Computer Methods in Applied Mechanics and Engineering 196 (35) (2007) 3381-3399.

[3] H. P. Bui, S. Tomar, S. P. A. Bordas, Real-time error control for surgical simulation, in: M. Brieu, S. Bordas, E. Leriche, S. Sherwin (Eds.), EUROMECH Colloquium 595, Biomechanics and computer assisted surgery meets medical reality, European Mechanics Society, Ecole Centrale Lille, Villeneuve dAscq, France, 2017. URL http://orbilu.uni.lu/handle/10993/29846

[4] D. Pham, C. Xu, J. Prince, Current methods in medical image segmentation 1, Annual review of biomedical engineering 2 (1) (2000) 315-337.

[5] S. Klein, U. van der Heide, I. Lips, M. van Vulpen, M. Staring, J. Pluim, Automatic segmentation of the prostate in $3 \mathrm{~d} \mathrm{mr}$ images by atlas matching using localized mutual information, Medical physics 35 (4) (2008) $1407-1417$.

[6] K. Miller, Computational biomechanics for patient-specific applications, Annals of biomedical engineering 44 (1) (2016) 1-2.

[7] M. Viceconti, L. Bellingeri, L. Cristofolini, A. Toni, A comparative study on different methods of automatic mesh generation of human femurs, Medical engineering \& physics 20 (1) (1998) 1-10. 
[8] M. Ferrant, A. Nabavi, B. Macq, F. A. Jolesz, R. Kikinis, S. Warfield, Registration of 3-d intraoperative mr images of the brain using a finiteelement biomechanical model, IEEE transactions on medical imaging 20 (12) (2001) 1384-1397.

[9] T. Belytschko, C. Parimi, N. Moës, N. Sukumar, S. Usui, Structured extended finite element methods for solids defined by implicit surfaces, International journal for numerical methods in engineering 56 (4) (2003) 609-635.

[10] V. Nguyen, T. Rabczuk, S. Bordas, M. Duflot, Meshless methods: A review and computer implementation aspects, Mathematics and Computers in Simulation 79 (3) (2008) 763 - 813. doi:http://doi.org/ $10.1016 / \mathrm{j} . \mathrm{matcom} .2008 .01 .003$.

[11] N. Stergiopulos, D. Young, T. Rogge, Computer simulation of arterial flow with applications to arterial and aortic stenoses, Journal of Biomechanics 25 (12) (1992) 1477 - 1488. doi:10.1016/0021-9290(92) 90060-E.

[12] S. Cotin, H. Delingette, N. Ayache, Real-time elastic deformations of soft tissues for surgery simulation, IEEE Transactions on Visualization and Computer Graphics 5 (1) (1999) 62-73. doi:10.1109/2945. 764872 .

[13] G. A. Holzapfel, R. W. Ogden, Biomechanical Modelling at the Molecular, Cellular and Tissue Levels, Springer, 2009. doi:10.1007/ 978-3-211-95875-9.

[14] Y. Payan, Soft Tissue Biomechanical Modeling for Computer Assisted Surgery, Springer, 2012. doi:10.1007/978-3-642-29014-5.

[15] L. Geris, D. Gomez-Cabrero, Uncertainty in Biology, Springer, 2016. doi:10.1007/978-3-319-21296-8.

[16] K. Miller, K. Chinzei, Mechanical properties of brain tissue in tension, Journal of Biomechanics 35 (4) (2002) 483 - 490. doi:http://dx.doi. org/10.1016/S0021-9290(01)00234-2.

[17] K. M. Labus, C. M. Puttlitz, An anisotropic hyperelastic constitutive model of brain white matter in biaxial tension and structura-mechanical relationships, Journal of the Mechanical Behavior of Biomedical Materials 62 (2016) 195-208. doi:10.1016/j.jmbbm.2016.05.003. 
[18] S. Chatelin, A. Constantinesco, R. Willinger, Fifty years of brain tissue mechanical testing: From in vitro to in vivo investigations, Biorheology 47 (2010) 255-276. doi:10.3233/BIR-2010-0576.

[19] K. Laksari, M. Shafieian, K. Darvish, Constitutive model for brain tissue under finite compression, Journal of Biomechanics 45 (2012) 642646. doi:10.1016/j.jbiomech.2011.12.023.

[20] L. Angela Mihai, L. Chin, P. Janmey, A. Goriely, A comparison of hyperelastic constitutive models applicable to brain and fat tissues, Journal of The Royal Society Interface 12 (110). doi:10.1098/rsif. 2015.0486 .

[21] R. de Rooij, E. Kuhl, Constitutive modeling of brain tissue: Current perspectives, Appl. Mech. Rev 68 (1). doi:10.1115/1.4032436.

[22] A. Goriely, S. Budday, E. Kuhl, Chapter two - neuromechanics: From neurons to brain, Vol. 48 of Advances in Applied Mechanics, Elsevier, 2015, pp. 79 - 139. doi:http://doi.org/10.1016/bs .aams.2015.10. 002 .

[23] G. Joldes, A. Wittek, K. Miller, Real-time nonlinear finite element computations on $\{$ GPU $\}$-application to neurosurgical simulation, Computer Methods in Applied Mechanics and Engineering 199 (49-52) (2010) 3305-3314. doi:http://doi.org/10.1016/j.cma.2010.06.037.

[24] C. A. Caflisch, Monte carlo and quasi -monte carlo methods, Acta numerica 7 (1998) 1-49.

[25] R. Ghanem, P. Spanos, Stochastic finite elements : a spectral approach, Dover publications 2003, Mineola, New York, 1991. doi: 10.1007/978-1-4612-3094-6.

[26] G. H. Matthies, Stochastic finite elements: Computational approaches to stochastic partial differential equations, Journal of Applied Mathematics and Mechanics 88 (2008) 849-873. doi:10.1002/zamm. 200800095 .

[27] L. Giraldi, D. Liu, H. G. Matthies, A. Nouy, To be or not to be intrusive? the solution of parametric and stochastic equations - the "plain vanilla" galerkin case, SIAM Journal on Scientific Computing 36 (2014) 2720-2744. doi:10.1137/140969063. 
[28] C. Dopico-González, A. New, M. Browne, Probabilistic finite element analysis of the uncemented hip replacementeffect of femur characteristics and implant design geometry, Journal of Biomechanics 43 (3) (2010) 512 - 520. doi:http://dx.doi.org/10.1016/j.jbiomech.2009.09. 039 .

[29] J. Biehler, M. W. Gee, W. A. Wall, Towards efficient uncertainty quantification in complex and large-scale biomechanical problems based on a bayesian multi-fidelity scheme, Biomechanics and Modeling in Mechanobiology 14 (3) (2015) 489-513. doi:10.1007/ s10237-014-0618-0.

URL http://dx.doi .org/10.1007/s10237-014-0618-0

[30] P. J. Laz, M. Browne, A review of probabilistic analysis in orthopaedic biomechanics, Journal of engineering in medecine 224 (2010) 927-943. doi:10.1243/09544119JEIM739.

[31] R. Plantefève, I. Peterlik, H. Courtecuisse, R. Trivisonne, J. Radoux, S. Cotin, Atlas-based transfer of boundary conditions for biomechanical simulation, Med Image Comput Comput Assist Interv. 17 (2014) 33-40.

[32] P. Risholm, A. Fedorov, J. Pursley, K. Tuncali, R. Cormack, W. Wells, Probabilistic non-rigid registration of prostate images: modeling and quantify uncertainty, IEEE Int Symp Biomed Imaging. (2011) 553556doi:10.1109/ISBI.2011.5872467.

[33] P. S. Mohan, P. B. Nair, A. J. Keane, Stochastic projection schemes for deterministic linear elliptic partial differential equations on random domains, Int. J. Numer. Meth. Engng 85 (2011) 874-895. doi:10. $1002 / \mathrm{nme} .3004$.

[34] F. Lindgren, H. Rue, J. Lindström, An explicit link between gaussian fields and gaussian markov random fields: the stochastic partial differential equation approach, Journal of the Royal Statistical Society: Series B (Statistical Methodology) 73 (4) (2011) 423-498. doi:10.1111/j.1467-9868.2011.00777.x.

[35] R. J. Adler, J. Taylor, Random fields and geometry, SpringerVerlagdoi:10.1007/978-0-387-48116-6.

[36] Y. Cao, M. Y. Hussaini, T. A. Zhang, Exploitation of sensitivity derivates for improving sampling methods, AIAA Journal 42 (2) (2004) 815-822. doi:10.2514/1.2820 
[37] Y. Liu, M. Y. Hussaini, G. Ökten, Optimization of a monte carlo variance reduction method based on sensitivity derivatives, Applied Numerical Mathematics 72 (Complete) (2013) 160-171. doi:10.1016/j. apnum.2013.06.005.

[38] P. Hauseux, J. S. Hale, S. P. Bordas, Solving the stochastic burgers equation with a sensitivity derivative-driven monte carlo method. URL https://doi.org/10.6084/m9.figshare.3561306

[39] P. Hauseux, J. S. Hale, S. P. Bordas, Accelerating monte carlo estimation with derivatives of high-level finite element models, Computer Methods in Applied Mechanics and Engineering 318 (2017) 917-936. doi:http://dx.doi.org/10.1016/j.cma.2017.01.041.

[40] M. Alnaes, J. Blechta, J. Hake, J. A., B. Kehlet, A. Logg, C. Richardson, J. Ring, M. Rognes, G. Wells, The fenics project version 1.5, Archive of Numerical Software 3 (100). doi:10.11588/ans.2015.100. 20553.

[41] A. Logg, G. Wells, Dolfin: Automated finite element computing, ACM Trans. Math. Softw. 37 (2) (2010) 20:1-20:28. doi:10.1145/1731022. 1731030 .

URL http://doi.acm.org/10.1145/1731022.1731030

[42] C. P. Robert, G. Casella, Monte Carlo Statistical Methods (Springer Texts in Statistics), Springer-Verlag New York, Inc., Secaucus, NJ, USA, 2005.

[43] P. E. Farrell, D. A. Ham, S. W. Funke, M. E. Rognes, Automated derivation of the adjoint of high-level transient finite element programs, SIAM Journal on Scientific Computing 35 (4) (2013) C369-C393. doi: $10.1137 / 120873558$.

[44] J. Feinberg, H. Langtangen, Chaospy: An open source tool for designing methods of uncertainty quantification, J. Comput. Science 11 (2015) 46-57. doi:10.1016/j.jocs.2015.08.008.

[45] I. Sobol, Global sensitivity indices for nonlinear mathematical models and their monte carlo estimates, Mathematics and Computers in Simulation 55 (13) (2001) 271 - 280, the Second \{IMACS $\}$ Seminar on Monte Carlo Methods. doi:http://dx.doi.org/10.1016/S0378-4754(00) 00270-6. 
[46] A. Saltelli, P. Annoni, I. Azzini, F. Campolongo, M. Ratto, S. Tarantola, Variance based sensitivity analysis of model output. design and estimator for the total sensitivity index, Computer Physics Communications 181 (2) (2010) 259 - 270. doi:http://dx.doi.org/10.1016/ j.cpc.2009.09.018.

[47] M. Giles, Multilevel monte carlo methods, Acta Numerica 24 (2015) 259-328. doi:10.1017/S096249291500001X.

[48] I. M. Sobol', Uniformly distributed sequences with an additional uniform property, USRR Computational Mathematics and Mathematical Physics 16 (1977) 236-242.

[49] W. Nowak, S. Tenkleve, O. A. Cirpka, Efficient computation of linearized cross-covariance and auto-covariance matrices of interdependent quantities, Mathematical Geology 35 (1) (2003) 53-66. doi: 10.1023/A: 1022365112368 .

[50] A. Logg, K. A. Mardal, G. N. Wells (Eds.), Automated Solution of Differential Equations by the Finite Element Method, Vol. 84 of Lecture Notes in Computational Science and Engineering, Springer, 2012. doi: 10.1007/978-3-642-23099-8.

[51] G. Holzapfel, R. Ogden, Constitutive modelling of passive myocardium: a structurally based framework for material characterization, Philosophical Transactions of the Royal Society of London A: Mathematical, Physical and Engineering Sciences 367 (1902) (2009) 3445-3475. doi:10.1098/rsta.2009.0091.

[52] F. Pérez, B. Granger, IPython: a system for interactive scientific computing, Computing in Science and Engineering 9 (3) (2007) 21-29. doi:10.1109/MCSE.2007.53.

[53] P. Hauseux, J. S. Hale, S. Cotin, S. P. Bordas, Solving a stochastic hyperelasticity equation with a sensitivity derivative-driven monte carlo method.

URL https://doi.org/10.6084/m9.figshare.4900298

[54] A. Valencia, B. Blas, J. H. Ortega, Modeling of brain shift phenomenon for different craniotomies and solid models, Journal of Applied Mathematics (2012) 1-21doi:10.1155/2012/409127. 
[55] S. Pezzuto, D. Ambrosi, A. Quarteroni, An orthotropic activestrain model for the myocardium mechanics and its numerical approximation, European Journal of Mechanics - A/Solids 48 (2014) 83 - 96, frontiers in Finite-Deformation Electromechanics. doi:http://dx.doi. org $/ 10.1016 / j$.euromechsol.2014.03.006

[56] S. Balay, S. Abhyankar, M. Adams, J. Brown, P. Brune, K. Buschelman, L. Dalcin, V. Eijkhout, W. Gropp, D. Kaushik, M. Knepley, L. Curfman, K. Rupp, B. Smith, S. Zampini, H. Zhang, H. Zhang, PETSc users manual, Tech. Rep. ANL-95/11 - Revision 3.7, Argonne National Laboratory (2016).

URL http://www.mcs.anl.gov/petsc

[57] R. Falgout, U. Yang, hypre: A Library of High Performance Preconditioners, in: P. M. A. Sloot, A. G. Hoekstra, C. J. K. Tan, J. J. Dongarra (Eds.), Computational Science ICCS 2002, no. 2331 in Lecture Notes in Computer Science, Springer Berlin Heidelberg, 2002, pp. 632-641. doi:10.1007/3-540-47789-6\_66.

[58] E. Chamberland, A. Fortin, M. Fortin, Comparison of the performance of some finite element discretizations for large deformation elasticity problems, Computers and Structures 88 (1112) (2010) $664-673$. doi: http://dx.doi.org/10.1016/j.compstruc.2010.02.007.

[59] A. Goriely, M. G. D. Geers, G. A. Holzapfel, J. Jayamohan, A. Jérusalem, S. Sivaloganathan, W. Squier, J. van Dommelen, S. Waters, E. Kuhl, Mechanics of the brain: perspectives, challenges, and opportunities, Biomechanics and Modeling in Mechanobiology 14 (5) (2015) 931-965. doi:10.1007/s10237-015-0662-4.

[60] Y. Feng, R. J. Okamoto, R. Namani, G. M. Genin, P. V. Bayly, Measurements of mechanical anisotropy in brain tissue and implications for transversely isotropic material models of white matter, Journal of the Mechanical Behavior of Biomedical Materials 23 (Supplement C) (2013) $117-132$

[61] E. K. Zander, Tensor approximation methods for stochastic problems, Shaker, 2012.

[62] B. Sudret, Global sensitivity analysis using polynomial chaos expansions., Reliability Engineering and System Safety 93 (7) (2008) 964-979. doi:10.1016/j.ress.2007.04.002. 
[63] N. Wiener, The homogeneous chaos, American Journal of Mathematics 60 (1938) 897-936. doi:10.2307/2371268.

[64] P. Malliavin, Stochastic analysis, Grundlehren der mathematischen Wissenschaften, Springer, Berlin, New York, 1997.

[65] H. Rappel, L. A. A. Beex, S. P. A. Bordas, Bayesian inference to identify parameters in viscoelasticity, Mechanics of Time-Dependent Materials (2017).

URL https://doi.org/10.1007/s11043-017-9361-0 